\title{
Weighted Multimodel Predictive Function Control for Automatic Train Operation System
}

\author{
Shuhuan Wen, ${ }^{1,2}$ Jingwei Yang, ${ }^{1}$ Ahmad B. Rad, ${ }^{3}$ Shengyong Chen, ${ }^{4}$ and Pengcheng Hao ${ }^{1}$ \\ ${ }^{1}$ Key Laboratory of Industrial Computer Control Engineering of Hebei Province, Yanshan University, Qinhuangdao 066004, China \\ ${ }^{2}$ State Key Laboratory of Rail Traffic Control and Safety, Beijing Jiaotong University, Beijing 100044, China \\ ${ }^{3}$ School of Engineering Science, Simon Fraser University, 250-13450, 102 Avenue, Surrey, BC, Canada V3T 0A3 \\ ${ }^{4}$ College of Computer Science and Technology, Zhejiang University of Technology, Hangzhou 310023, China
}

Correspondence should be addressed to Shengyong Chen; csy@zjut.edu.cn

Received 23 September 2013; Accepted 28 November 2013; Published 3 February 2014

Academic Editor: M. Montaz Ali

Copyright (C) 2014 Shuhuan Wen et al. This is an open access article distributed under the Creative Commons Attribution License, which permits unrestricted use, distribution, and reproduction in any medium, provided the original work is properly cited.

\begin{abstract}
Train operation is a complex nonlinear process; it is difficult to establish accurate mathematical model. In this paper, we design ATO speed controller based on the input and output data of the train operation. The method combines multimodeling with predictive functional control according to complicated nonlinear characteristics of the train operation. Firstly, we cluster the data sample by using fuzzy-c means algorithm. Secondly, we identify parameter of cluster model by using recursive least square algorithm with forgetting factor and then establish the local set of models of the process of train operation. Then at each sample time, we can obtain the global predictive model about the system based on the weighted indicators by designing a kind of weighting algorithm with error compensation. Thus, the predictive functional controller is designed to control the speed of the train. Finally, the simulation results demonstrate the effectiveness of the proposed algorithm.
\end{abstract}

\section{Introduction}

With rapid expansion of the nationwide metropolitan population, people put forward higher requirement for the speed and quality of the urban railway traffic train. The Automatic Train Control (ATC) system, as an important subsystem of the automatic train control system, can replace the drivers to realize automatic train operation. On the basis of ensuring safety and punctuality, it can furthest implement the demand of energy conservation and improve passengers comfort. It is the prospective development direction of the railway train.

Train operation is a complex nonlinear process; it is difficult to establish accurate mathematical model because of nonlinearities and complex operating environments such as ramps, curves, and tunnels. So it increases difficulties to realize the ATO system. Based on the input and output data, people will apply some normal nonlinear system identification methods, like Hammerstein model structure [1], Wiener model structure [2], and so forth. But the model structure cannot reveal global performance of the complicated system.
Multimodel method based on decomposition combination rule can provide an effective way for the modeling and control problem of complicated nonlinear system. Multiple linear local models are used to replace the complicated controlled object to simplify the nonlinear system structure. And the control algorithm based on linear model can be applied conveniently to nonlinear control system.

For multimodel control system, firstly we should build local linear model set of the nonlinear system. The common method is to linearize the nonlinear system near the equilibrium point to get local model set [3]. However, it is difficult to confirm the equilibrium point and model because of the complication of train operation. Based on the input and output data of nonlinear systems, cluster analysis divides the data according to some kind of similarity criterion. The method that combines cluster analysis with traditional identification provides an effective way to establish the local model set of nonlinear system.

Predictive functional control is the third generation of model-predictive control algorithm. It not only has the main 
features of model-predictive control, but also owns its unique advantages. The introduction of base function can make the control input structure more clear, and obviously reduces online computation. This paper proposes a method based on the actual train operation. It combines cluster multimodel weighted modeling with predictive functional control algorithm to design ATO speed controller. Firstly, we use fuzzy c-means (FCM) clustering algorithm with recursive least square algorithm of forgetting factor to establish the local model set of nonlinear operation process. In order to improve the performance of control system, we design a kind of weighted algorithm with error compensation. The global model will be achieved through weighted combination of local models, and then we can design predictive functional controller.

\section{Problem Information}

According to the train dynamics, the dynamic equations can be described as follows [4]:

$$
\begin{gathered}
\frac{d s(t)}{d t}=v(t), \\
\frac{d v(t)}{d t}=u(t)-w(v(t))-g(s(t)), \\
w(v(t))=a v(t)^{2}+b v(t)+c, \\
g(s(t))=l \sin (\alpha(s)),
\end{gathered}
$$

where $s$ is the train position coordinate, $v$ is the velocity of train, $u$ is the traction force or breaking force, $w(v)$ is motion resistance related to velocity, $g(s)$ is external resistance caused by the slope, curve, and wind, $l$ is a constant, and $\alpha(s)$ is slope degree of $s$ point.

In (1), we can see that train operation is a nonlinear process. As the speed increases, the nonlinearity will get stronger, and the operation speed will be easily affected by external resistance. The purpose of this paper is to design reasonable ATO speed control algorithm to make train operation as objective speed curve and distance curve. The objective speed curve and distance curve are obtained by computing optimal operation figure.

\section{The Establishment of Multimodels Set}

Cluster analysis is a kind of data mining technology, which is widely researched and applied. It can divide physical or abstract data into groups in accordance to similarity degree. This feature provides an effective way to divide models into the groups of multimodel modeling. In the field of process modeling, cluster multimodel modeling adequately excavates relationships between the data based on the input and output data. It divides the data into different features groups reasonably. In recent years, multimodel modeling method based on clustering is widely used and a large number of cluster algorithms emerge. The fuzzy c-means clustering algorithm is a simple and effective clustering method.

The FCM is an unsupervised clustering algorithm. A clustering problem can be expressed as follows.
Let $X=\left\{x_{1}, \ldots, x_{n}\right\}$ be a set of given data, where each data point $x_{k}(k=1, \ldots, n)$ is a vector in $\mathfrak{R}^{p}$ and $n$ is the number of sample data. Its aim is to find the membership matrix $R=\left[r_{i j}\right]_{c \times n}$ and the cluster centers of sample data set $O=$ $\left[o_{1}, o_{2}, \ldots, o_{c}\right]$ that minimize a dissimilarity function. When the clustering number is given, FCM can be described as a mathematical programming problem [5]:

$$
\begin{array}{ll}
\min & J_{m}(R, o)=\sum_{j=1}^{n} \sum_{i=1}^{c} r_{i j}^{m}\left\|x_{j}-o_{i}\right\|^{2} \\
\text { s.t. } & \sum_{i=1}^{c} r_{i j}=1, \quad 1 \leq j \leq n \\
& r_{i j} \in[0,1], \quad 1 \leq j \leq n, 1 \leq i \leq c \\
& 0<\sum_{j=1}^{n} r_{i j}<n,
\end{array}
$$

where $r_{i j}$ represents the membership of $x_{j}$ in the cluster $i$, $x_{j} \in \mathfrak{R}^{p}$ is the $j$ th measured data, $o_{i} \in \mathfrak{R}^{p}$ is the $i$ th center of the cluster, $\|*\|$ denotes the distance of the measured data from the cluster center which can be measured by means of Euclidean norm, $m$ is any real number larger than 1, and $m=2$ is used in this contribution. $r_{i j}$ and $o_{i}$ can be obtained from the paper [5].

In multimodel control, it is important to establish the multiple models set. Xie and Beni introduced a valid measure. The separation measure $V_{x b}$ is defined as in [6].

The optical cluster number is

$$
c_{\mathrm{opt}}=\arg \min _{c}\left(V_{x b}\right)
$$

In summary, the procedure for the fuzzy c-means algorithm is as follows.

Step 1. Collect the input and output data of training operation, and assume the maximum cluster number is 10 . We apply Xie-Beni cluster validity index to assure the optical cluster number $c$. Initialize $R=R_{0}$, preset the index weight $m$, and $\varepsilon>0$.

Step 2. At $k$ th iteration, compute the cluster centers $o_{i}^{k}$ where $r_{i j} \in R^{k}$ ( $o_{i}^{k}$ and $R^{k}$, are resp., the cluster centers of sample data set and the membership matrix of the $k$ th iteration).

Step 3. Update $R^{k+1}$ using $r_{i j}\left(R^{k+1}\right.$ is the membership matrix of the $(k+1)$ th iteration).

Step 4. If $\left\|R^{k+1}-R^{k}\right\|>\varepsilon$, then $k=k+1$; go to Step 2, otherwise stop.

For each cluster set, the parameters of sublinear model are identified by using the forgetting factor recursive least squares (RLS) algorithm. To design GPC controller conveniently, we 


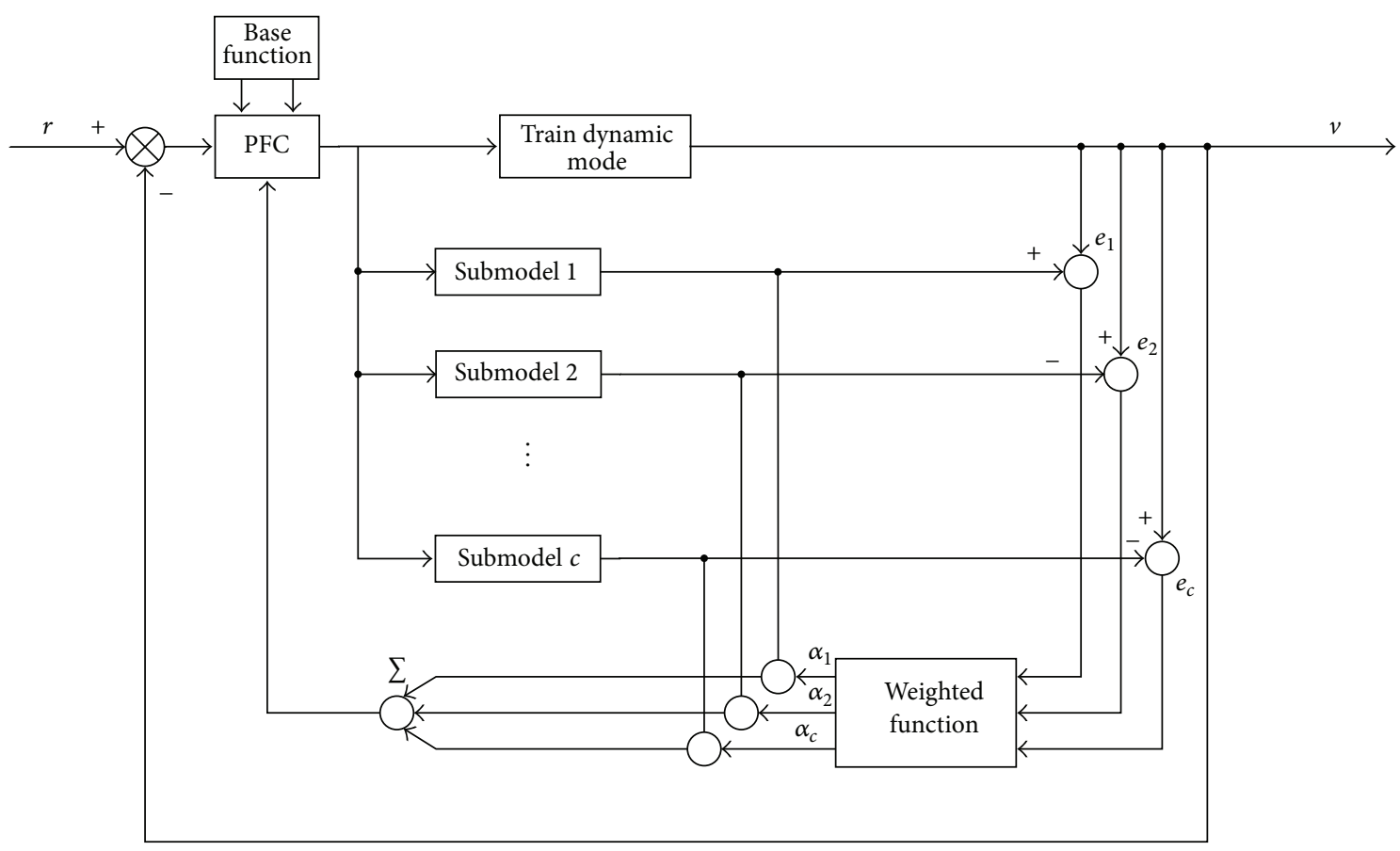

FIGURE 1: The structure of multimodel weighted predictive function control for train.

choose SISO (single-input single-output) Controlled AutoRegressive Integrated Moving Average (CARIMA) model. So the sublinear model is described as follows [7]:

$$
\begin{gathered}
A\left(z^{-1}\right) y(t)=B\left(z^{-1}\right) u(t-1)+\frac{\zeta(t)}{\Delta}, \\
A\left(z^{-1}\right)=1+a_{1} z^{-1}+\cdots+a_{n_{a}} z^{-n_{a}}, \\
\operatorname{deg} A\left(z^{-1}\right)=n_{a}, \\
B\left(z^{-1}\right)=b_{0}+b_{1} z^{-1}+\cdots+b_{n_{b}} z^{-n_{b}}, \\
\operatorname{deg} B\left(z^{-1}\right)=n_{b},
\end{gathered}
$$

where $u(t)$ is the control input and $y(t)$ is the measured variable or output; in this paper $y(t)=v(t)$, and $\zeta(t)$ is the white noise in which the mean value is zero; $n_{a}$ and $n_{b}$ are the orders of output and input, respectively. $\Delta=1-z^{-1}$ is the differencing operator; $A\left(z^{-1}\right), B\left(z^{-1}\right)$, and $C\left(z^{-1}\right)$ are polynomials with the backward shift operator $z^{-1}$. Among them, the first several elements of the polynomial $B\left(z^{-1}\right)$ can be zero to express the corresponding delay numbers. The sublinear model can be identified by using the form of regression equation.

\section{Multimodel Predictive Function Controller}

4.1. The Structure of Multimodel Weighted Predictive Function. The structure of multimodel weighted predictive function is shown in Figure 1. In Figure 1, the submodels are obtained by clustering and identification algorithm. At each control time, we compare the error of real output and the output of submodel $i$. According to the matching degree, the corresponding weights are assigned to each submodel by weighted function. The bigger weight value represents the smaller mismatch of submodel.

4.2. Multimodel Weighted Strategy. Multimodel control algorithm based on weighted method can make full advantage of system information of each local model and describe the dynamics of nonlinear system more accurately at the overlap of multiple subspace. The key question is to find the appropriate weighted strategy to enable local model to approach nonlinear dynamics effectively. Based on the multimodel integration strategy proposed in the literature [8], this paper proposes an improved multimodel weighted strategy with error compensation.

Define $e_{i}(t)=y(t)-\hat{y}_{i}(t)$. The expression denotes the error between real output and the output of the submodel $i$ at time $t$. The average matching error between submodel $i$ and system at time $t$ is shown as follows:

$$
m e_{i}(t)=\frac{e_{i}(t)+\sum_{j=1}^{L} \eta^{L} e(t-j)}{L+1},
$$

where $L$ denotes the error length in the past time at the average error computation and $\eta$ denotes the forgetting degree of the past error.

The performance index based on model matching degree is defined as follows:

$$
\widetilde{J}_{i}(t)=\frac{\left|e_{i}(t)-m e_{i}(t)\right|+\sum_{j=1}^{L} \eta^{L}\left|e_{i}(t-j)-m e_{i}(t)\right|}{L+1} .
$$


As (7) shows, the weight of submodel $i$ is

$$
\alpha_{i}(t)=\frac{\widetilde{J}_{i}(t)^{-1}}{\sum_{i=1}^{c} \widetilde{J}_{i}(t)^{-1}},
$$

where $c$ is the number of local models.

After adding weighted value, the global predictive model is

$$
\begin{aligned}
\widehat{y}(t)= & \sum_{i=1}^{c} \alpha_{i} a_{1}^{i} y(t-1)+\sum_{i=1}^{c} \alpha_{i} a_{2}^{i} y(t-2) \\
& +\cdots+\sum_{i=1}^{c} \alpha_{i} a_{n_{a}}^{i} y\left(t-n_{a}\right) \\
& +\sum_{i=1}^{c} \alpha_{i} b_{0}^{i} u(t-1)+\sum_{i=1}^{c} \alpha_{i} b_{1}^{i} u(t-2) \\
& +\cdots+\sum_{i=1}^{c} \alpha_{i} b_{n_{b}}^{i} u\left(t-n_{b}-1\right) .
\end{aligned}
$$

4.3. Predictive Function Controller Design. Because of its convenient derivation and strong robustness, predictive function control absorbs many scholars. At the present stage, the research of PFC mostly aims at first-order system or firstorder plus pure delay system. Because the first-order system, first-order plus pure delay system, and second-order system can be described by CARIMA model, it is more general to study the PFC algorithm based on CARIMA model. In this paper, we study the predictive function control based on CARIMA model; the structure of predictive model is shown as (4).

Designing ATO system needs to guarantee the travelling comfort, and it is closely related to operation velocity. So the control input should decrease large fluctuation in designing ATO system. For this purpose, we add summation form of control increment to optimized performance indicator; optimized performance is shown as follows [9]:

$$
\min J=\min \left[\left(Y_{b}-Y_{r}\right)^{T}\left(Y_{b}-Y_{r}\right)+\rho \Delta U^{T} \Delta U\right]
$$

where

$$
\begin{aligned}
Y_{b} & =\left[y_{b}(t+1), y_{b}(t+2), \ldots, y_{b}\left(t+N_{1}\right)\right]^{T} ; \\
Y_{r} & =\left[y_{r}(t+1), y_{r}(t+2), \ldots, y_{r}\left(t+N_{1}\right)\right]^{T} ; \\
\Delta U & =\left[\Delta u(t), \Delta u(t+1), \ldots, \Delta u\left(t+N_{u}-1\right)\right]^{T} .
\end{aligned}
$$

In predictive function control, control input is regarded as a linear combination of base function which is given advance [9]; generally ramp function and step functions can satisfy most of the control requirements. So in this paper, control input is regarded as weight combination of two base function proposed before; the form is shown as follows:

$$
u(t+i)=\mu_{1}+\mu_{2} i, \quad\left(i=0,1, \ldots, N_{u}-1\right) .
$$

Equation (12) can be rewritten as

$$
U=\left[\begin{array}{c}
u(t) \\
u(t+1) \\
\vdots \\
u\left(t+N_{u}-1\right)
\end{array}\right]=\left[\begin{array}{cc}
1 & 0 \\
1 & 1 \\
\vdots & \vdots \\
1 & N_{u}-1
\end{array}\right]\left[\begin{array}{l}
\mu_{1} \\
\mu_{2}
\end{array}\right]=C_{1} \bar{\mu}
$$

where

$$
C_{1}=\left[\begin{array}{cc}
1 & 0 \\
1 & 1 \\
\vdots & \vdots \\
1 & N_{u}-1
\end{array}\right]_{N_{u} \times 2}, \quad \bar{\mu}=\left[\begin{array}{l}
\mu_{1} \\
\mu_{2}
\end{array}\right] .
$$

In PFC, the output $y(t)$ of predictive model is composed of free response $y_{f}(t)$ and forced response $y_{p}(t)$; the form is shown as follows [9]:

$$
y(t)=y_{p}(t)+y_{f}(t)
$$

So the predictive output of PFC is

$$
y(t+i)=y_{p}(t+i)+y_{f}(t+i), \quad\left(i=1,2, \ldots, N_{1}\right) .
$$

As for the model free response output $Y_{f}=\left[y_{f}(t+1)\right.$, $\left.y_{f}(t+2), \ldots, y_{f}\left(t+N_{1}\right)\right]^{T}$, Zhang and Wangquanling, and so forth proposed a kind of recursive deprivation [10]:

$$
\begin{aligned}
y_{f}(t+1 \mid t)= & \sum_{j=1}^{n_{a}}\left(-a_{j}\right) y^{\prime}(t+i-j) \\
& +\sum_{j=1}^{n_{b}} b_{j} u^{\prime}(t+i-j), \quad i=1,2, \ldots, N_{1},
\end{aligned}
$$

where

$$
\begin{gathered}
y^{\prime}(t+i-j)= \begin{cases}y(t+i-j), & i<j+1, \\
y_{f}(t+i-j \mid t), & i \geq j+1,\end{cases} \\
u^{\prime}(t+i-j)= \begin{cases}u(t+i-j), & i<j, \\
0, & i \geq j .\end{cases}
\end{gathered}
$$

As for the model forced response output $Y_{p}=\left[y_{p}(t+1)\right.$, $\left.y_{p}(t+2), \ldots, y_{p}\left(t+N_{1}\right)\right]^{T}$ can be derived as follows:

$$
y_{p}(t+i \mid t)=\sum_{j=1}^{i} r_{j} u(t+i-j), \quad\left(i=1,2, \ldots, N_{1}\right),
$$

where

$$
\begin{gathered}
r_{1}=b_{1}, \\
r_{j}=\sum_{k=1}^{\min \left(j-1, n_{a}\right)}\left(-a_{k}\right) r_{j-k}+b_{j}, \quad 2 \leq j \leq n_{b}, \\
r_{j}=\sum_{k=1}^{\min \left(j-1, n_{a}\right)}\left(-a_{k}\right) r_{j-k}, \quad n_{b}<j \leq N_{1} .
\end{gathered}
$$


From (13) and (19), it can derived that

$$
\begin{aligned}
Y_{p} & =\left[\begin{array}{c}
y_{p}(t+1) \\
y_{p}(t+2) \\
\vdots \\
y_{p}\left(t+N_{1}\right)
\end{array}\right] \\
& =\left[\begin{array}{cccc}
r_{1} & 0 & \cdots & 0 \\
r_{2} & r_{1} & \cdots & 0 \\
\vdots & \vdots & \ddots & \vdots \\
r_{N_{1}} & r_{N_{1}-1} & \cdots & r_{1}
\end{array}\right]\left[\begin{array}{cc}
1 & 0 \\
1 & 1 \\
\vdots & \vdots \\
1 & N_{1}-1
\end{array}\right]\left[\begin{array}{l}
\mu_{1} \\
\mu_{2}
\end{array}\right] \\
= & C_{2} C_{3} \bar{\mu},
\end{aligned}
$$

where

$$
C_{2}=\left[\begin{array}{cccc}
r_{1} & 0 & \cdots & 0 \\
r_{2} & r_{1} & \cdots & 0 \\
\vdots & \vdots & \ddots & \vdots \\
r_{N_{1}} & r_{N_{1}-1} & \cdots & r_{1}
\end{array}\right] ; \quad C_{3}=\left[\begin{array}{cc}
1 & 0 \\
1 & 1 \\
\vdots & \vdots \\
1 & N_{1}-1
\end{array}\right] .
$$

To reduce the effect caused by model mismatch, error correction is used to compensate the predictive output.

Consider

$$
y_{b}(t+i)=y(t+i)+e(t+i) .
$$

Predictive error is described as the following form:

$$
e(t+i)=y(t)-y_{m}(t), \quad i=0,1, \ldots, N_{1},
$$

where $y(t)$ is the output of the predictive model and $y_{m}(t)$ is the actual output of the system.

So

$$
Y_{b}=Y_{p}+Y_{f}+C_{4} e(t)=C_{2} C_{3} \bar{\mu}+Y_{f}+C_{4} e(t),
$$

where $C_{4}=[1 ; 1 ; \ldots ; 1]_{N_{1} \times 1}$.

Because $\Delta u(t)=u(t)-u(t-1)$, from (13), we can get that

$$
\begin{aligned}
& \Delta U=\left[\begin{array}{c}
\Delta u(t) \\
\Delta u(t+1) \\
\vdots \\
\Delta u\left(t+N_{u}-1\right)
\end{array}\right] \\
& =\left[\begin{array}{c}
u(t)-u(t-1) \\
u(t+1)-u(t) \\
\vdots \\
u\left(t+N_{u}-1\right)-u\left(t+N_{u}-2\right)
\end{array}\right] \\
& =\left[\begin{array}{cccc}
1 & 0 & \cdots & 0 \\
-1 & 1 & \cdots & 0 \\
\vdots & \vdots & \cdots & 0 \\
0 & 0 & \cdots & 1
\end{array}\right]\left[\begin{array}{c}
u(t) \\
u(t+1) \\
\vdots \\
u\left(t+N_{u}-1\right)
\end{array}\right]-\left[\begin{array}{c}
u(t-1) \\
0 \\
\vdots \\
0
\end{array}\right] \\
& =C_{5} U-U_{0}=C_{5} C_{1} \bar{\mu}-U_{0} \text {, }
\end{aligned}
$$

where

$$
C_{5}=\left[\begin{array}{cccc}
1 & 0 & \cdots & 0 \\
-1 & 1 & \cdots & 0 \\
\vdots & \vdots & \cdots & 0 \\
0 & 0 & \cdots & 1
\end{array}\right]_{N_{u} \times N_{u}}, \quad U_{0}=\left[\begin{array}{c}
u(t-1) \\
0 \\
\vdots \\
0
\end{array}\right]_{N_{u} \times 1}
$$

Substituting (23) and (26) into (10) and letting $\partial J / \partial \bar{\mu}=0$, we can get that

$$
\begin{aligned}
\bar{\mu}= & {\left[\left(C_{2} C_{3}\right)^{T}\left(C_{2} C_{3}\right)+\rho\left(C_{5} C_{1}\right)^{T}\left(C_{5} C_{1}\right)\right]^{-1} } \\
& \times\left[\left(C_{2} C_{3}\right)^{T}\left(Y_{r}-Y_{f}-C_{4} e(t)\right)+\rho\left(C_{5} C_{1}\right)^{T} U_{0}\right] .
\end{aligned}
$$

Let $C_{2} C_{3}=C_{6}, C_{5} C_{1}=C_{7}$; we can get that

$$
\bar{\mu}=\left(C_{6}^{T} C_{6}+\rho C_{7}^{T} C_{7}\right)^{-1}\left[C_{6}^{T}\left(Y_{r}-Y_{f}-C_{4} e(t)\right)+\rho C_{7}^{T} U_{0}\right] .
$$

From (12) and (29), we can get control subsequence of the future time. Predictive function control retains the rolling optimization strategy and just applies the current control input to system. So the current control input of predictive function control is

$$
u(t)=\mu_{1}+\mu_{2} \times 0=\mu_{1}=[1,0] \bar{\mu} .
$$

\section{Simulation Results and Discussions}

A concrete train operation system [11] is used to test the performance of the proposed algorithm. We consider a 17285.5 meters long trail line. Because curves and tunnel external force will convert to ramp force under a certain condition, we just consider a length of ramp force in simulation. It is defined as follows:

$$
g(s(t))= \begin{cases}\left(\frac{0.2}{1000}\right) \times s(t)-1.0, & 5000<s(t) \leq 6000 \\ 0.2, & 6000<s(t) \leq 7000 \\ -\left(\frac{0.2}{800}\right) \times s(t)+1.95, & 7000<s(t) \leq 7800 .\end{cases}
$$

The unit basic resistance model is determined empirically from empirical formula which is derived by experiments. In this paper we choose unit basic resistance model of Shenzhen metro line 3. It is defined as follows:

$$
w_{0}(v(t))=0.00675 v^{2}(t)+0.394 v(t)+20.89 .
$$

In the operation process of the train, one of energysaving control scheme is to keep a constant speed in the cruise stage, reducing unnecessary braking and acceleration. This simulation imitates the constant-speed cruise motion of 
TABLE 1: The result of cluster.

\begin{tabular}{cccc}
\hline$C$ & $V_{x b}$ & $C$ & $V_{x b}$ \\
\hline 2 & 0.1344 & 7 & 0.0431 \\
3 & 0.0798 & 8 & 0.0410 \\
4 & 0.0451 & 9 & 0.1505 \\
5 & 0.0221 & 10 & 0.1602 \\
6 & 0.0816 & - & - \\
\hline
\end{tabular}

the rail train. We can obtain the ATO speed by composite computation:

$$
v_{d}(t)= \begin{cases}0.75 t, & 0 \leq t \leq 40 \\ 30, & 40<t \leq 560 \\ -0.35 t+226, & 560<t \leq 600 \\ -0.7 t+436, & 600<t \leq 610 \\ -t+619, & 610<t \leq 619\end{cases}
$$

The distance profile in the simulation is as follows:

$$
s_{d}(t)= \begin{cases}0.375 t^{2}, & 0 \leq t \leq 40 \\ 600+30(t-40), & 40<t \leq 560 \\ -0.175 t^{2}+226 t-55480, & 560<t \leq 600 \\ -0.35 t^{2}+436 t-118480, & 600<t \leq 610 \\ -0.5 t^{2}+619 t-174295, & 610<t \leq 619\end{cases}
$$

First of all, we obtain the input and output data of train operation based on dynamic model. According to the fuzzy cmeans cluster algorithm and Xie-Beni cluster valid measure, we can obtain the optimal number of submodels. The result of clustering is shown in Table 1.

In Table $1, C$ denotes the number of cluster; $V_{x b}$ denotes the value of cluster valid measure. When the cluster number is 5, the value of $V_{x b}$ is the minimum. So the optimal number of cluster is 5 . According to the clustering set and forgetting factor recursive least squares identification algorithm, the submodels set can be defined as follows.

Submodel 1:

$$
y(t)-0.9515 y(t-1)=0.0375 u(t-1)+\zeta(t)
$$

Submodel 2:

$$
y(t)-0.9790 y(t-1)=0.0150 u(t-1)+\zeta(t) ;
$$

Submodel 3:

$$
y(t)-0.9891 y(t-1)=0.0020 u(t-1)+\zeta(t) ;
$$

Submodel 4:

$$
y(t)-0.9748 y(t-1)=0.0161 u(t-1)+\zeta(t) ;
$$

Submodel 5:

$$
y(t)-0.9902 y(t-1)=0.0051 u(t-1)+\zeta(t) .
$$

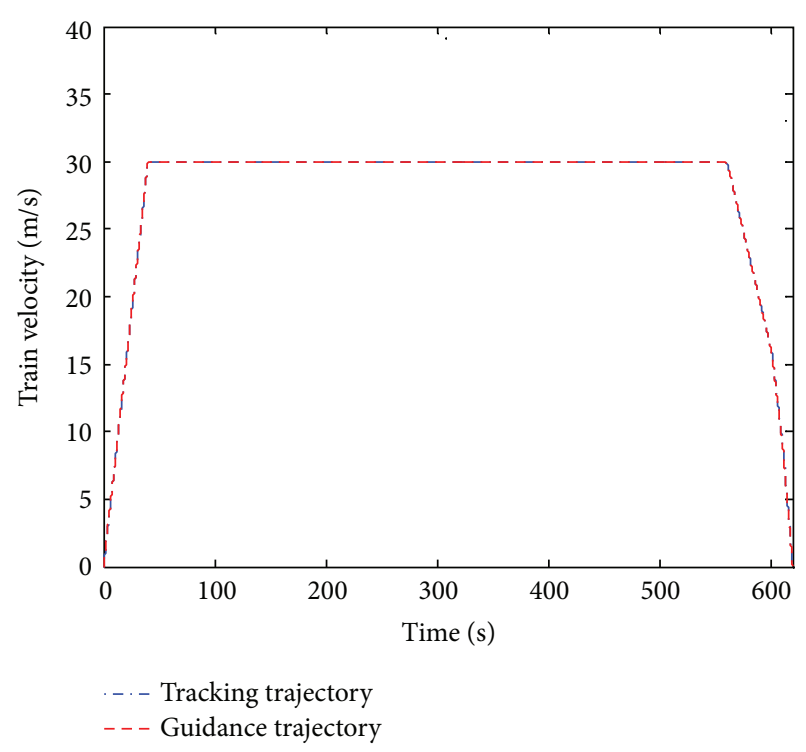

FIGURE 2: Train velocity tracking curve (this paper).

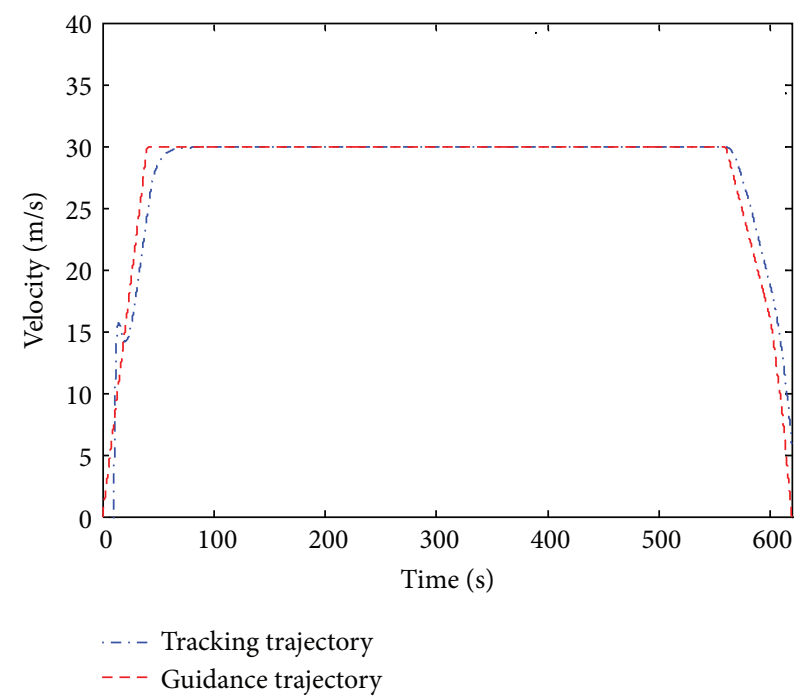

FIgURE 3: Train velocity tracking curve (single model PFC).

In controller designing, we define $L=2, \eta=0.9$ according to the weighted scheme; the parameters of predictive function controller are $N_{1}=5$ and $N_{u}=2$. The algorithm proposed in this paper is compared with single-model PFC algorithm and the result is shown in Figures 2 and 3.

From Figures 2 and 3, we can see that the proposed scheme can track the ATO speed profile precisely and achieve the tracking task. But at some local working points, the singlemodel predictive function control algorithm cannot track the objective curve precisely. The reason is that single-model predictive function control algorithm cannot describe the global performance of nonlinear train operation. The multimodel control algorithm has better control performance, and can describe global state of nonlinear system. 


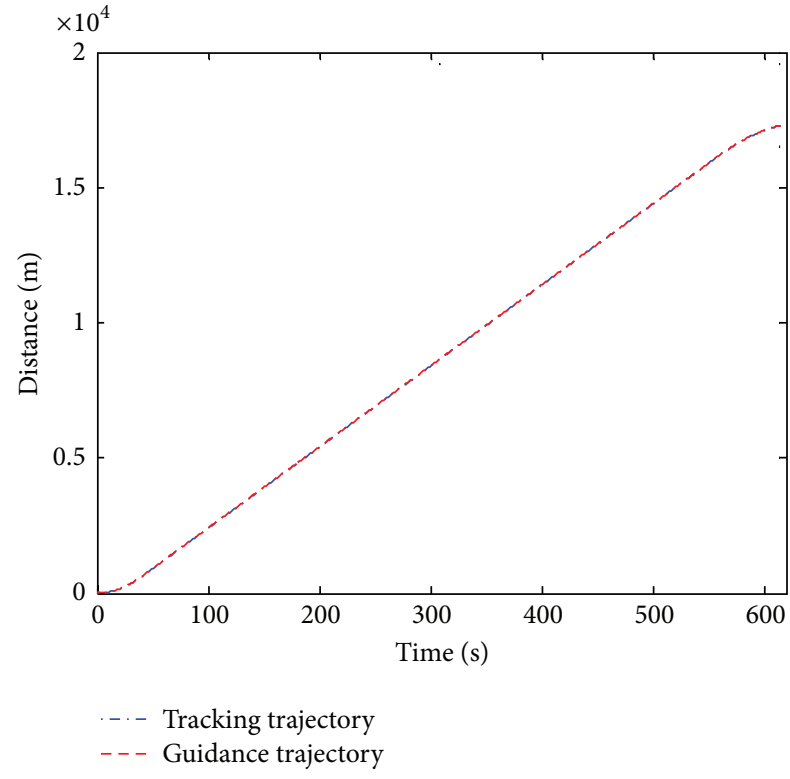

FIGURE 4: Train distance tracking curve (this paper).

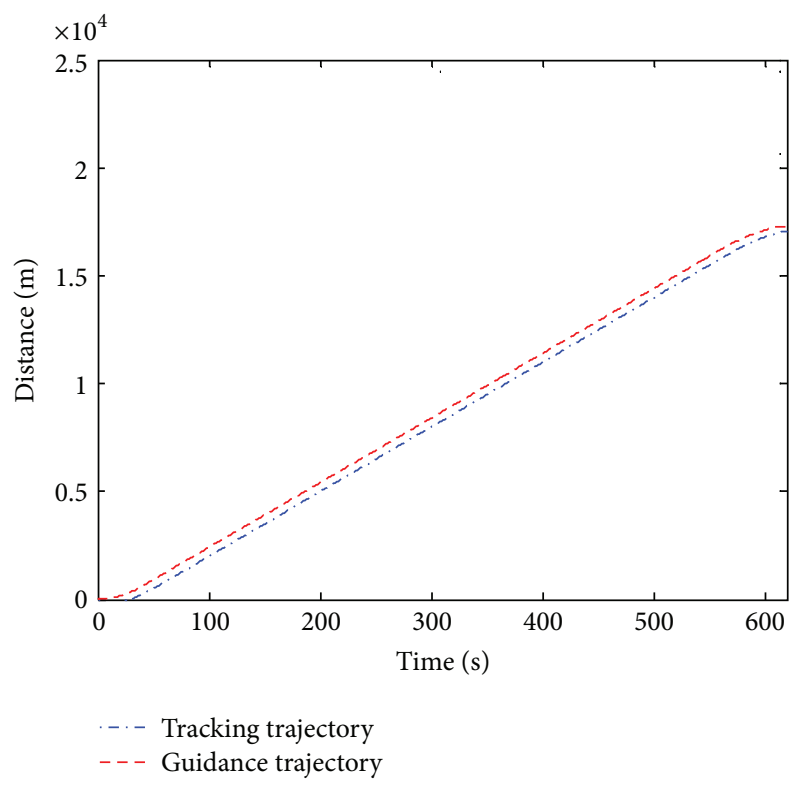

FIGURE 5: Train distance tracking curve (single model PFC).

In Figures 4 and 5, we can see that the algorithm of the ATO speed controller proposed in this paper can make the curve almost match together. And the results meet the accurate requirement of the stopping. Because at some local working points the single-model PFC algorithm cannot describe the operation character precisely, the distance trajectory cannot track the given trajectory.

Figure 6 shows the tracking situation of train acceleration and the given acceleration under the control of multimodel predictive function control. Figure 7 shows the part weighted changing curves of local model in multimodel control. The results show that more placidly the stable weights changing

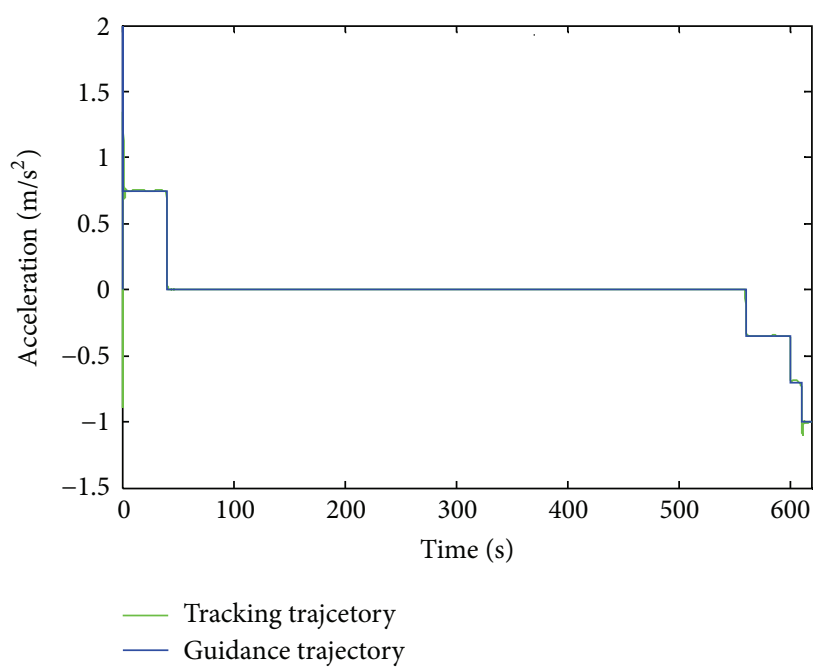

FIgURE 6: Train acceleration tracking curve (this paper).

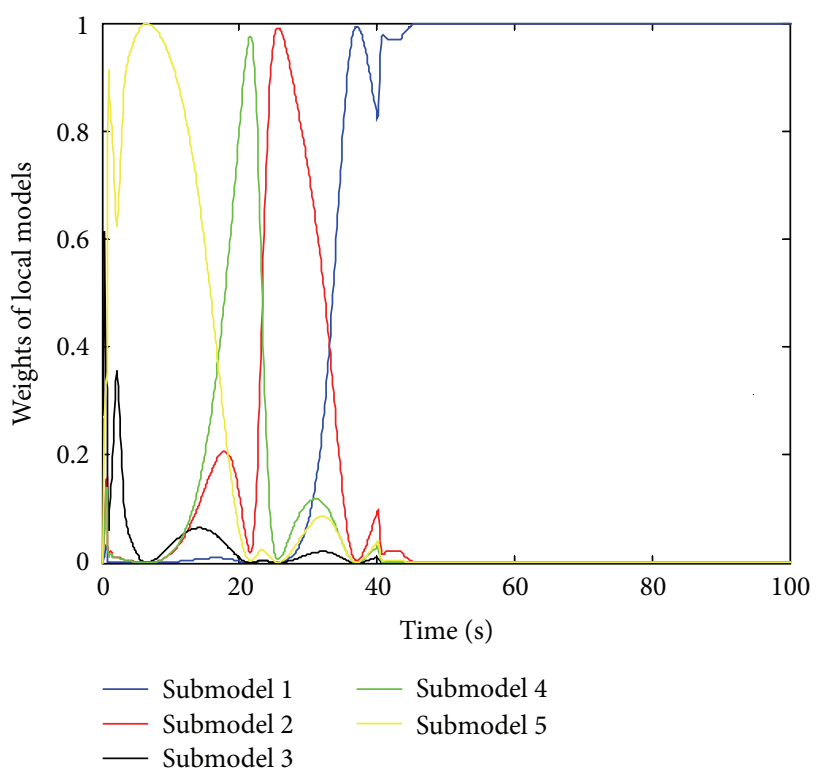

Figure 7: The figure of weight change of local model.

can assure the transformation of global model. Then the acceleration will not vibrate largely when the models transform.

\section{Conclusions}

In this paper, we studied the speed controller of ATO system by combining clustering multimodel weighted modeling method with predictive function control algorithm. The proposed algorithm solved the modeling and control problem of nonlinear train operation system. Firstly, we used clustering and identification algorithm to build local model set of train operation process. At each sample time, weighted scheme with error compensation is combined with every local model according to the respective weight to obtain global model. 
After meeting linear requirement, we can design predictive function controller. Finally, the simulation results are provided to show the effectiveness of the proposed algorithm.

\section{Conflict of Interests}

The authors declare that there is no conflict of interests regarding the publication of this paper.

\section{Acknowledgments}

This work is supported by the State Key Laboratory of Rail Traffic Control and Safety (Contract no. RCS2011K007), Beijing Jiaotong University and the Hebei Province Natural Science Foundation under project Grant no. F2014203095, the Young Teacher of Yanshan University under the project no. 13LGA007, National Natural Science Foundation of China (61325019, 61173096, and R1110679), and International Science \& Technology Cooperation Program of China (S2014GAT030).

\section{References}

[1] F. Ding and T. Chen, "Identification of Hammerstein nonlinear ARMAX systems," Automatica, vol. 41, no. 9, pp. 1479-1489, 2005.

[2] G. Shafiee, M. M. Arefi, M. R. Jahed-Motlagh, and A. A. Jalali, "Nonlinear predictive control of a polymerization reactor based on piecewise linear Wiener model," Chemical Engineering Journal, vol. 143, no. 1-3, pp. 282-292, 2008.

[3] X. Yugeng and W. Fan, "Nonlinear multi-model predictive control," Acta Automatica Sinica, vol. 22, no. 4, pp. 456-460, 1996.

[4] Y. Wang and Z. S. Hou, "Terminal iterative learning control for station stop control of a train," in Proceedings of the Symposium on Learning Control at IEEE CDC, vol. 6, pp. 3119-3124, Shanghai, China, December 2009.

[5] T. Velmurugan and T. Santhanam, "Performance evaluation of K-means and fuzzy C-means clustering algorithms for statistical distributions of input data points," European Journal of Scientific Research, vol. 46, no. 3, pp. 320-330, 2010.

[6] C.-H. Chou, M.-C. Su, and E. Lai, "A new cluster validity measure and its application to image compression," Pattern Analysis and Applications, vol. 7, no. 2, pp. 205-220, 2004.

[7] D. W. Clarke, C. Mohtadi, and P. S. Tuffs, "Generalized predictive control-part I. The basic algorithm," Automatica, vol. 23, no. 2, pp. 137-148, 1987.

[8] P. P. Bonissone, F. Xue, and R. Subbu, "Fast meta-models for local fusion of multiple predictive models," Applied Soft Computing Journal, vol. 11, no. 2, pp. 1529-1539, 2011.

[9] S. Wen, "A fast algorithm for adaptive predictive function of Hénon chaotic system," Acta Physica Sinica, vol. 58, no. 8, pp. 5209-5213, 2009.

[10] Q. L. Zhang and S. Q. Wang, "Adaptive predictive function control using ARMAX models," Information and Control, vol. 29, no. 5, pp. 431-436, 2000.

[11] X. Dexin, "Choice of maximum running speed of vehicles for urban mass transit," Journal of Railway Engineering, vol. 2, no. 113, pp. 97-99, 2008. 


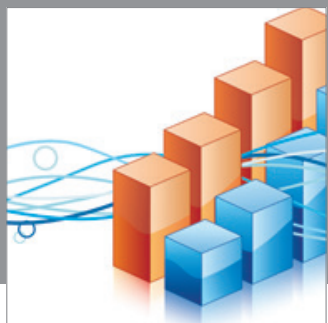

Advances in

Operations Research

mansans

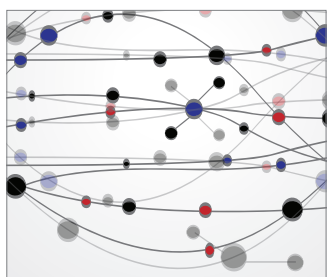

The Scientific World Journal
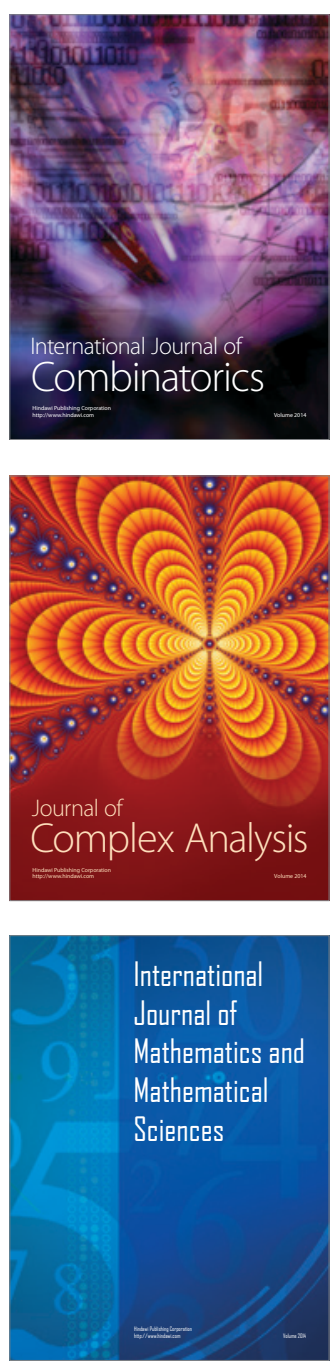
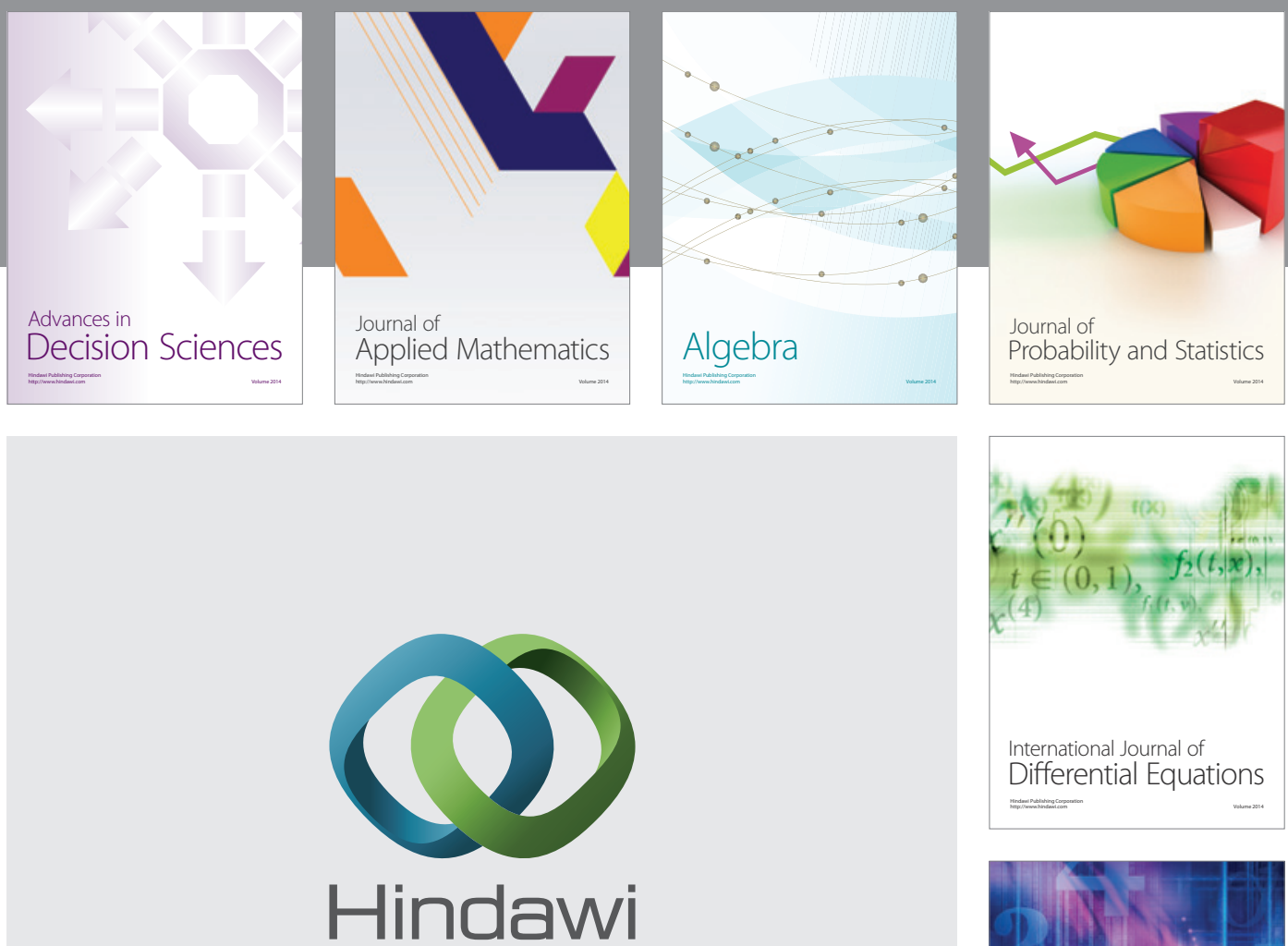

Submit your manuscripts at http://www.hindawi.com
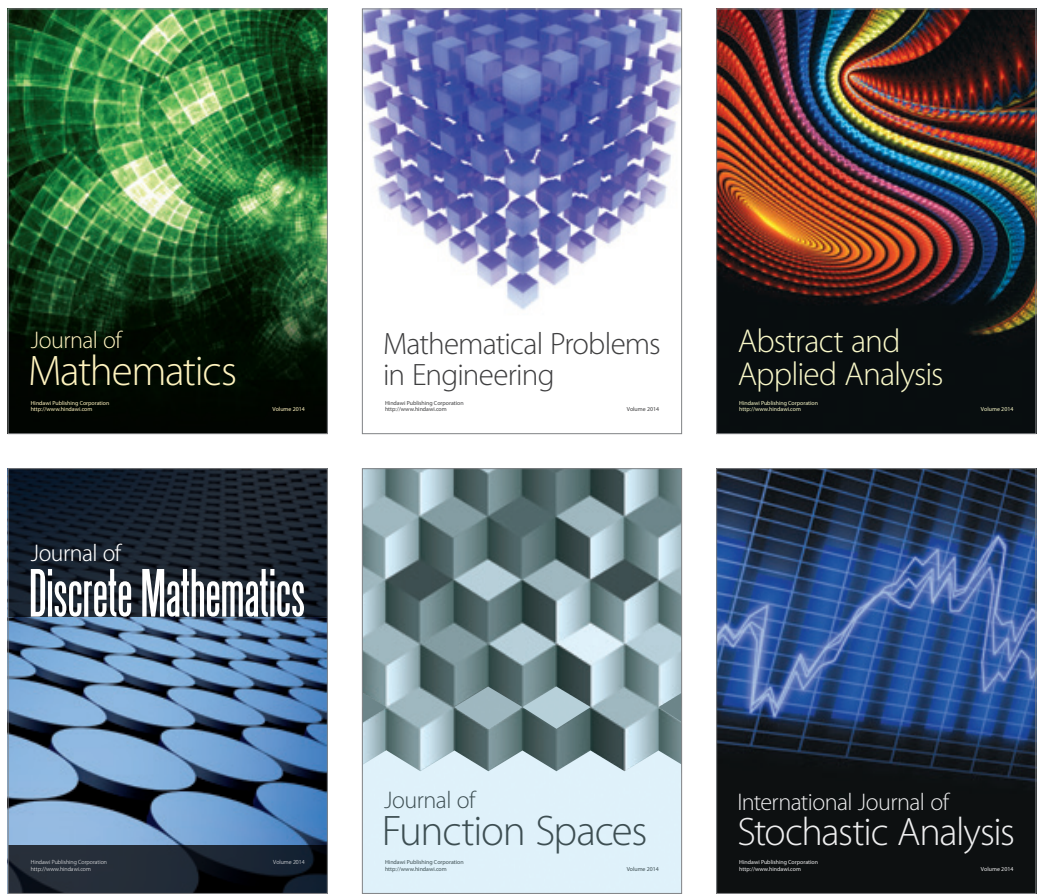

Journal of

Function Spaces

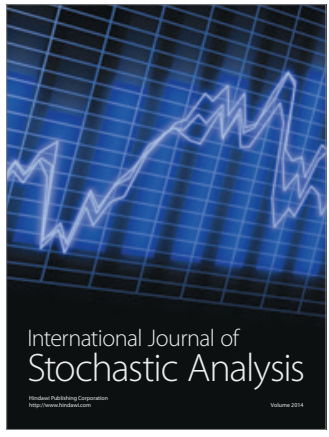

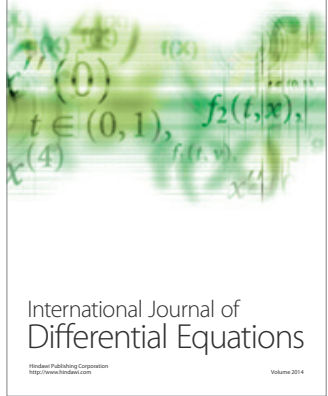
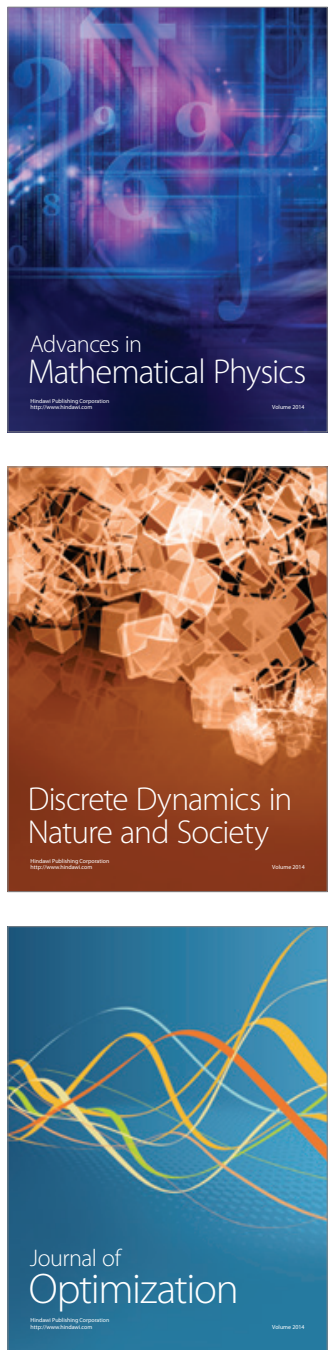